\title{
Characterization of Abrupt Heterojunctions in SiGe NW using Off-Axis Electron Holography
}

\author{
Zhaofeng Gan ${ }^{1}$, Daniel Perea ${ }^{2,3}$, S. Tom Picraux ${ }^{2}$, David J. Smith ${ }^{1}$ and Martha R. McCartney ${ }^{1}$ \\ 1. Department of Physics, Arizona State University, Tempe, AZ 85287. \\ 2. Center for Integrated Nanotechnologies, Los Alamos National Laboratory, Los Alamos, NM 87545. \\ 3. Environmental Molecular Sciences Laboratory, Pacific Northwest National Laboratory, Richland, WA \\ 99352.
}

Heterojunction SiGe nanowires (NWs) have important applications such as tunneling field effect transistors [1]. However, it is difficult to grow abrupt SiGe heterojunctions using the vapor-liquid-solid (VLS) growth method as well as make direct measurements of the built-in potential across the junction due to the nanoscale dimensions. Off-axis electron holography is an effective way to measure electrostatic potential with nanometer spatial resolution [2]. By using the reconstructed phase image, the projected potential distribution along the electron beam direction of the object can be retrieved. Here, we have used off-axis electron holography to measure the potential profile across $p$ - $n$ heterojunctions in SiGe NWs. The heterojunction NWs were grown using the VLS method on [111] Ge substrates using AuGa particles as catalysts [3]. The heterojunction morphology consists of a $\sim 4 \mu \mathrm{m}$ B-doped $\left(\sim 10^{17} \mathrm{~cm}^{-3}\right)$ Ge base and a $300 \mathrm{~nm}$ P-doped $\left(\sim 10^{18} \mathrm{~cm}^{-3}\right)$ Si tip. After growth, the NWs were transferred to carbon grids for TEM examination.

Figure 1a shows a TEM image of a typical SiGe NW with the AuGa catalyst still present at the tip. Darker contrast is observed at the Ge/Si interface and is likely due to lattice mismatch strain between Ge and Si. However, contrast at the heterojunction interface is not visible in the HAADF image in Figure 1b. Measurement of (111) lattice fringes across the SiGe heterojunction indicated that the lattice spacing changed from $0.327 \mathrm{~nm}$ Ge (111) to $0.314 \mathrm{~nm} \mathrm{Si(111)} \mathrm{with} \mathrm{a} \mathrm{spacing} \mathrm{less} \mathrm{than} \mathrm{Si}(111)$ in the middle region (not shown here). The cause of this smaller spacing is still unclear, but it might also be due to strain. Figure 2 shows the line profile of HAADF intensity, Ge EDX K edge and Si EDX K edge across the SiGe junction. These indicate that the composition changes from Ge to Si with a junction width of about $27 \mathrm{~nm}(10 \%-90 \%)$ for the wire with a diameter of $115 \mathrm{~nm}$. Figure 3 shows the phase image after reconstruction of the complex wave function from the hologram. In the phase profile along the long axis, there is a decrease of 0.5 radian, going from $105 \mathrm{~nm}$ to $180 \mathrm{~nm}$. At about $120 \mathrm{~nm}$, there is also a lower phase dip, which might be due to diffraction caused by strain at the interface. A closer look at the edge of the NW in the phase image also shows that the edge is at constant phase, or equipotential, across the $p$ - $n$ junction, which suggests that the potential is pinned at mid gap at the NW surface. Despite the complexity of the interface, the built-in potential of SiGe $p$ - $n$ junction is calculated to be $1.1 \mathrm{~V}$ from the far end of Ge and Si away from interface, using the values of $14.3 \mathrm{~V}$ and $12.1 \mathrm{~V}$ for the mean inner potential of $\mathrm{Ge}$ and $\mathrm{Si}$, respectively. This value is high compared to the estimated value of $0.4 \mathrm{~V}$, which is calculated based on the growth conditions and abrupt junction assumption, and might be affected by diffraction by the wire or surface pinning. Further experiments and simulations are in progress to improve the accuracy of the measurements [4].

\section{References}

[1] A. M. Ionescu and H. Riel, Nature 479 (2011), 329.

[2] M. R. McCartney and D. J. Smith, Ann. Rev. Mater. Res. 37 (2007), 729. 
[3] D. E. Perea, et al., Nano Lett. 11 (2011), 3117.

[4] The electron holography studies have been supported by DoE Grant DE-FG02-04ER46168. We gratefully acknowledge the use of facilities within the John M. Cowley Center for High Resolution Electron Microscopy at Arizona State University. We also acknowledge use of both the CINT and EMSL user facilities.

(a)

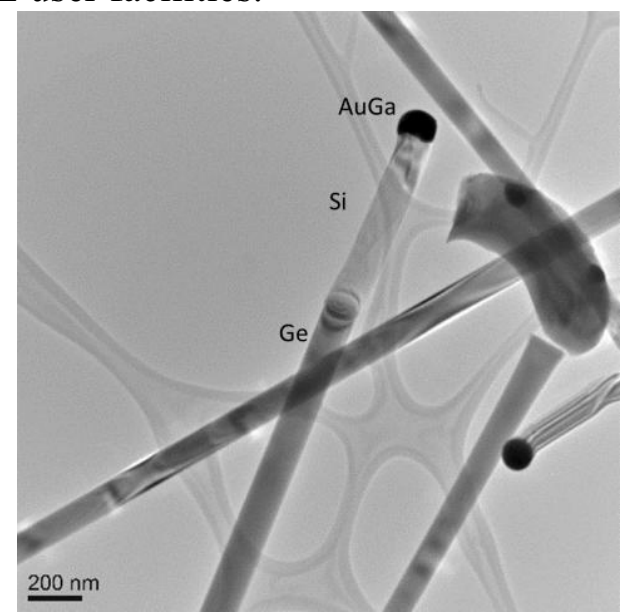

(b)

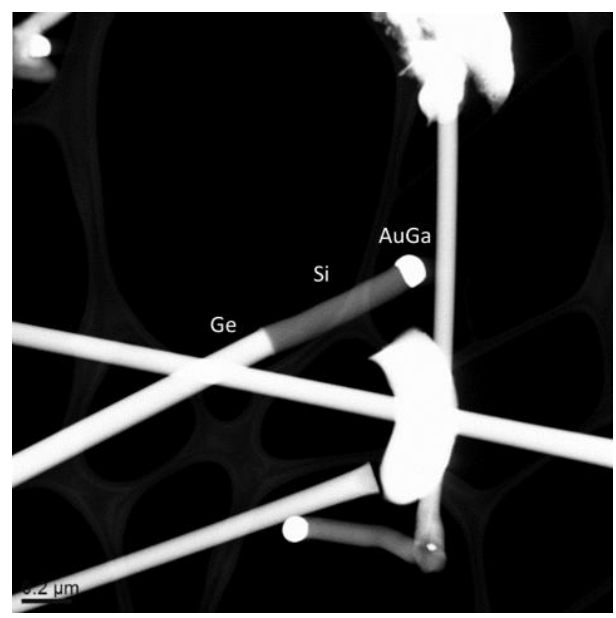

Figure 1. Low resolution TEM and HAADF images of SiGe NWs.

(a)

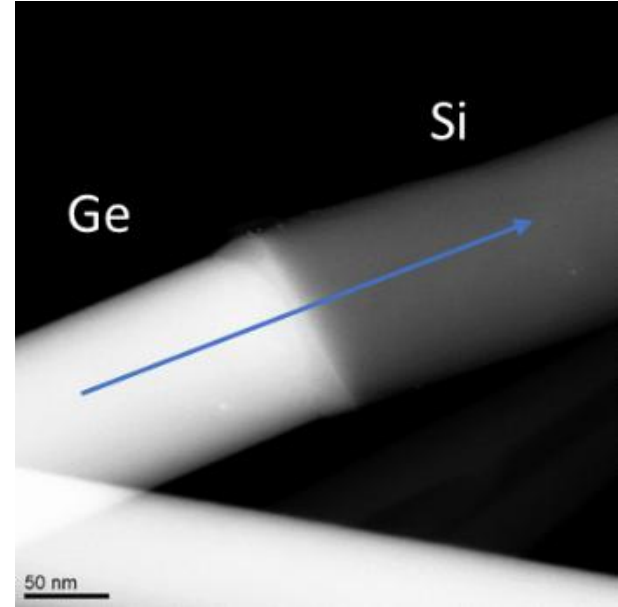

(b)

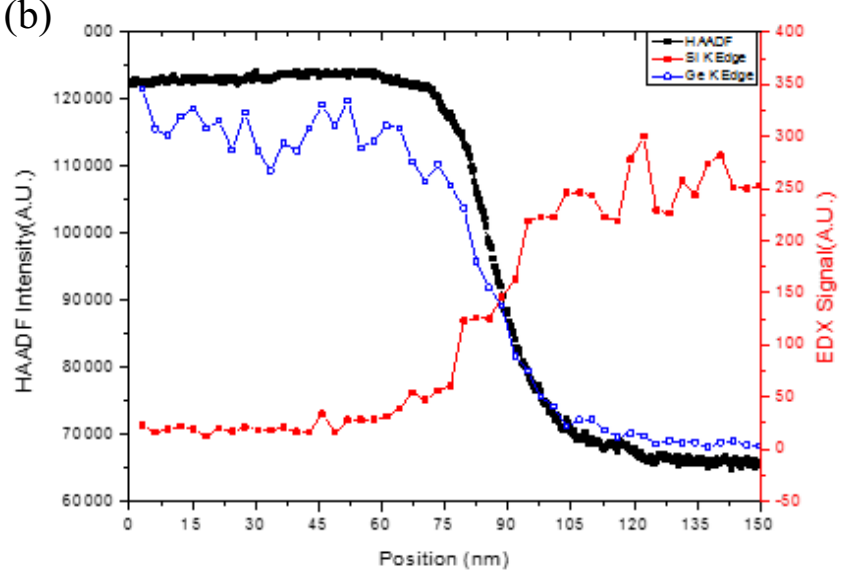

Figure 2. (a) HAADF image of SiGe heterojunction; (b) HAADF intensity and EDX line profiles along blue arrow.

(a)

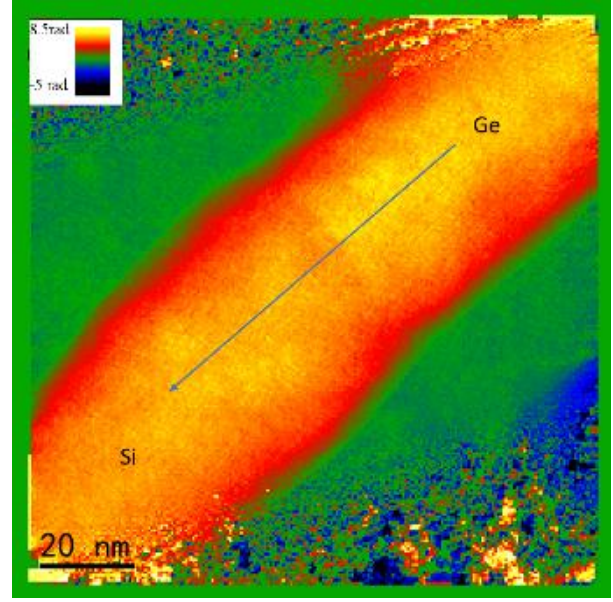

(b)

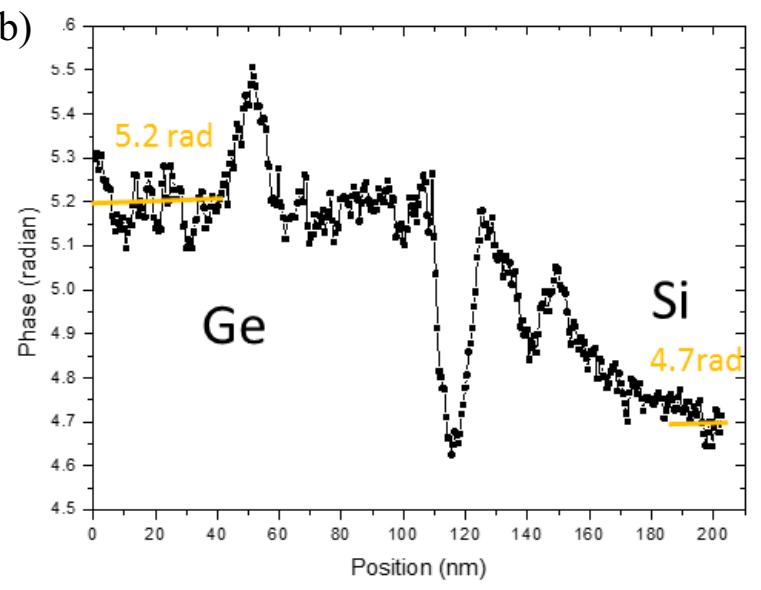

Figure 3. (a) Reconstructed phase image of SiGe heterojunction, and (b) phase profile along blue arrow. 